\title{
Structural design process and subsequent flight mechanical evaluation in preliminary aircraft design: demonstrated on passenger ride comfort assessment
}

\author{
Vikram Krishnamurthy $^{1}\left({ }^{\circledR} \cdot\right.$ Vega Handojo $^{2} \odot$
}

Received: 11 March 2020 / Revised: 11 February 2021 / Accepted: 31 March 2021 / Published online: 19 April 2021

(c) The Author(s) 2021

\begin{abstract}
New fuel-efficient aircraft designs have high aspect ratio wings. Consequently, those aircraft are more flexible. Additionally, load alleviation functions are implemented to reduce the structural loads, which results in further reductions of the structural stiffness. At the same time, the structural design impacts other disciplines in preliminary aircraft design, especially flight mechanics. For example, it is important to know how at that design stage such flexible aircraft with load alleviation affect passenger ride comfort in turbulent flight. For an efficient design process, it is essential to answer such questions with accurate multi-disciplinary tools and methods as early as possible to minimize development risk and avoid costly and time-consuming redesign loops. Current available tools and methods are not accurate enough for this task. To address this issue, the DLR MONA based design and the TUB flight mechanical assessment tool MITRA are linked to investigate the impact of the structural design on specific flight mechanical assessments such as passenger ride comfort. This is particularly interesting since the implemented load alleviation functions are designed to reduce loads, and not explicitly to improve passenger ride comfort. By conducting this assessment for a particular aircraft configuration, more insight into passenger ride comfort and the key contributors can be gained during preliminary design. This paper describes the combined toolchain and its application on a generic long-range reference aircraft to investigate the effects of load alleviation functions on passenger ride comfort and discusses the results.
\end{abstract}

Keywords Flight mechanics $\cdot$ Flight simulation $\cdot$ Ride comfort $\cdot$ Gust load alleviation $\cdot$ Structural optimization

\begin{tabular}{|c|c|c|c|}
\hline \multicolumn{2}{|c|}{ Abbreviations } & GLA & Gust load alleviation \\
\hline AFT & Most rear CG location & GLR & Generic long range \\
\hline CFD & Computer fluid dynamics & LAT & Lateral \\
\hline CS-25 & Certification specification for large aeroplanes & LON & Longitudinal \\
\hline DLM & Doublett-lattice method & LRA & Load reference axis \\
\hline DLR & German Aerospace Center & MITRA & Multiobjective Evaluation of Preliminary \\
\hline EOM & Equation of motion & & Aircraft Designs \\
\hline FCL & Flight control laws & MLA & Manoeuvre load alleviation \\
\hline FCS & Flight control system & MONA & ModGen Nastran (in-house design process \\
\hline \multirow{2}{*}{$\begin{array}{l}\text { FE } \\
\text { FWD }\end{array}$} & Finite element & & of DLR) \\
\hline & Most forward CG & MTOM & Maximum take-off mass \\
\hline \multirow{5}{*}{$\begin{array}{ll}\square \mathrm{Vi} \\
\mathrm{Kr}\end{array}$} & Krichnomurthy & OEM & Operating empty mass \\
\hline & murthy@tu-berlin.de & PSD & Power spectral density \\
\hline & 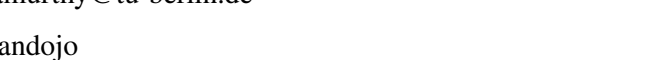 & RFA & Rational function approximation \\
\hline & andojo@dlr.de & RMS & Root mean square \\
\hline & & SOL & Solution sequence \\
\hline \multirow{2}{*}{$\begin{array}{l}\text { In: } \\
\text { Un }\end{array}$} & of Aeronautics and Astronautics, Technische & TAS & True airspeed \\
\hline & ität Berlin (TUB), Berlin, Germany & TUB & Technische Universität Berlin \\
\hline $\begin{array}{ll}2 & \text { Ins } \\
& \text { Gö }\end{array}$ & of Aeroelasticity, German Aerospace Center (DLR), & VLM & Vortex lattice method \\
\hline
\end{tabular}




\section{ZFM Zero fuel mass \\ c.g. Centre of gravity}

\section{Symbols}

\section{$D \quad$ Damping}

$D_{\text {lat }} \quad$ Discomfort due to lateral acceleration

$D_{\text {long }} \quad$ Discomfort due to longitudinal acceleration

$D_{\mathrm{LP}} \quad$ Discomfort due to long-pitch acceleration

$D_{\text {pitch }} \quad$ Discomfort due to pitch acceleration

$D_{\text {roll }} \quad$ Discomfort due to roll acceleration

$D_{\text {vert }} \quad$ Discomfort due to vertical acceleration

$D_{\text {VIB }} \quad$ Discomfort due to vibration

$D_{\mathrm{VLR}} \quad$ Discomfort due to vertical-lateral-roll acceleration

$T_{2} \quad$ Time to double the bank angle

$T_{\mathrm{R}} \quad$ Roll time constant

$V \quad$ Velocity

$V_{\mathrm{D}} \quad$ Dive speed

$a \quad$ Acceleration

$g \quad$ Gravitational acceleration

$q \quad$ Pitch rate

$w_{\text {wg }} \quad$ Vertical wind speed

$\Theta \quad$ Pitch angle

$\Phi \quad$ Bank angle

$\Psi \quad$ Yaw angle

$\alpha \quad$ Angle of attack

$\xi \quad$ Aileron deflection

$\omega_{0} \quad$ Natural frequency

\section{Introduction}

New fuel-efficient aircraft designs have high aspect ratio wings and lower structural masses, as currently shown with A330-800 and B777x, and are more flexible. Aircraft manufacturers strive to reduce structural loads by active load control which results in an additional reduction of the structural stiffness. Within the design process, there are strong interdependencies between each involved discipline due to its interdisciplinary nature. For an efficient process, it is essential to use accurate multi-domain tools and methods as early as possible to minimize development risks and avoid costly and time-consuming redesign loops.

One key design discipline which is significantly impacted by structural design choices and active load control is flight mechanics, for which one must conduct assessments regarding safety and performance continuously. In preliminary aircraft design, aircraft manufacturers currently use semiempirical evaluation methods, e.g. from Ref. [1]. They are not accurate enough to investigate the impact of coupling effects of advanced load design and flight mechanics.

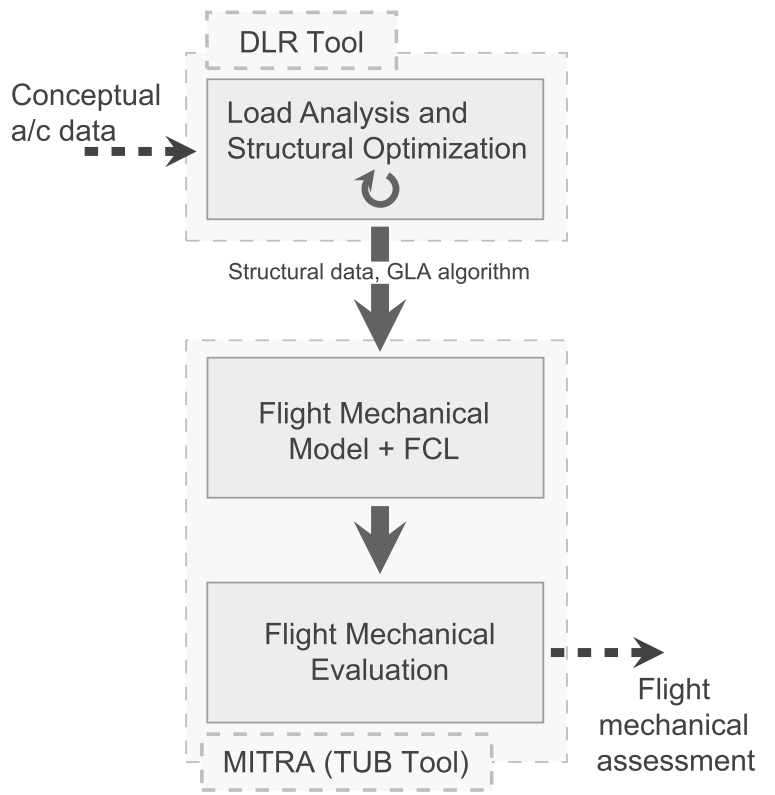

Fig. 1 DLR-TUB toolchain: structural design process with flight mechanical assessment

To address this issue, the German Aerospace Center (DLR) loads analysis and structural optimization process [2] and the flight mechanical assessment tool MITRA of Technische Universität Berlin (TUB) [3] are linked together for a seamless flight mechanical evaluation of structural design choices, see Fig. 1.

The iterative load analysis and subsequent structural optimization-based on the DLR MONA process-matches the aircraft structure with the design loads. MITRA uses the resulting data to automatically generate a flight mechanical model of the flexible aircraft and affiliated flight control laws (FCL) including gust load alleviation (GLA) for flight simulations in the time domain. It is then possible to mimic flight tests for automated flight mechanical evaluation.

This DLR-TUB toolchain allows simple flight mechanical assessments of advanced structural design choices as well as more complex ones, which previously were not possible in preliminary aircraft design. A desirable assessment is the estimation of passenger ride comfort for the acceptance of new flexible aircraft with active load control in turbulent flight. The results of the assessment is not apparent. For example, GLA might impact translational and angular accelerations acting on the passenger at certain frequencies resulting in decreased or increased passenger comfort. If any undesirable effects on passenger ride comfort can be identified early in preliminary aircraft design, they can be still mitigated with less costs than in later design stages. That is why this work demonstrates the DLR-TUB toolchain by investigating advanced structural design choices on passenger ride comfort. 


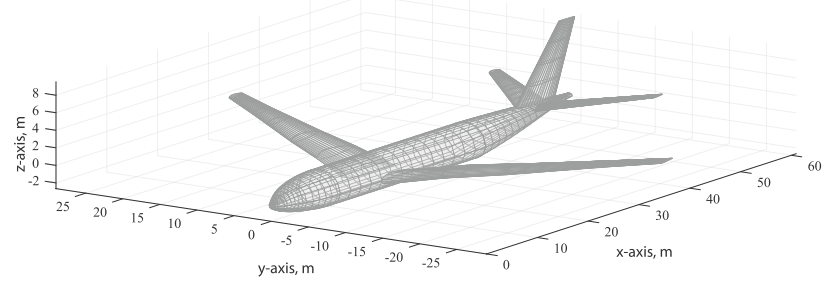

Fig. 2 A three dimensional model of the reference aircraft without engines and pods

Table 1 GLR configuration design parameters

\begin{tabular}{ll}
\hline Parameter & Value \\
\hline Wing area & $360 \mathrm{~m}^{2}$ \\
Wing span & $58 \mathrm{~m}$ \\
Mean aerodynamic chord & $7.2 \mathrm{~m}$ \\
Operating empty mass (OEM) & $120,000 \mathrm{~kg}$ \\
Maximum take off mass (MTOM) & $235,000 \mathrm{~kg}$ \\
Cruise Mach number & 0.82 \\
\hline
\end{tabular}

Table 2 Normalized frequencies of selected modes for different mass configurations

\begin{tabular}{lll}
\hline Normalized mode & OEM & MTOM \\
\hline First symmetric wing bending mode & 1.00 & 0.82 \\
Symmetric, vertical engine mode & 1.37 & 1.32 \\
Symmetric, lateral engine mode & 1.41 & 1.41 \\
First vertical fuselage bending mode & 1.80 & 1.53 \\
\hline
\end{tabular}

This toolchain and the subsequent assessment is demonstrated on a generic long-range reference (GLR) aircraft configuration designed for 250 passengers, see Sect. 2. It shall be noted that the outcome of this investigation is not only dependent on the structural design but also the implemented FCL and GLA. Due to the modular tool structure, other control configurations can be implemented and assessed using the same methods to find an optimal design.

\section{Model description}

The reference aircraft for the ride comfort assessment is the GLR configuration shown in Fig. 2. Table 1 lists its design parameters and Table 2 frequencies of selected modes for different mass configurations. All values in Table 2 are normalized to the first symmetric wing bending frequency of the operating empty mass (OEM) configuration. For the integration of the masses, the OEM consists of structural masses as well as secondary and operational masses (galley,

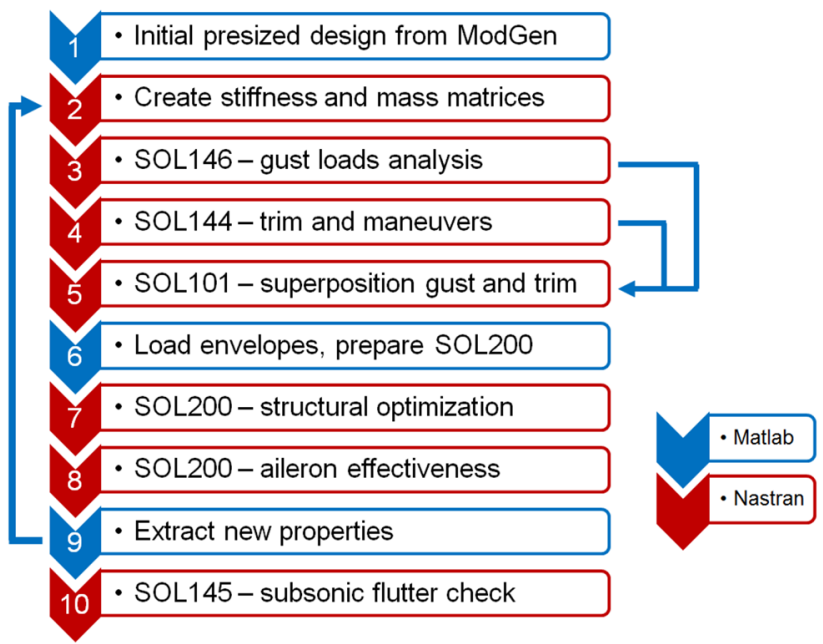

Fig. 3 Process of loads analysis and structural optimization

landing gear, crew etc.) located at the respective positions. For the condensation, the different masses are assigned to the nearest load reference axis (LRA) node and accumulated using ModGen, and the output is a single mass (CONM2) for the concerning LRA node. Further details can be found in Ref. [2].

\section{Design process with integrated load alleviation}

DLR developed the MONA process, see Ref. [2], to set up and optimize aeroelastic models in preliminary aircraft design. The name MONA stands for ModGen, a DLR inhouse program to generate models, and MSC.Nastran. The MONA process starts with a preliminary load estimation based on conceptual design methods, followed by a parameterized setup of simulation models and an optimization model. With these models, the MONA process conducts a load analysis and a subsequent structural optimization iteratively. The steps with the load analysis and structural optimization are extended and elaborated in more detail below.

\subsection{Process of loads analysis and structural optimization}

Figure 3 shows the MONA-based design process used to optimize the reference aircraft. SOL stands for the respective solution sequences of MSC.Nastran. With the pre-sized aircraft design resulting from ModGen, the stiffness and mass properties of the aircraft model are condensed onto the load reference axis (LRA) nodes to save computing time in the simulations. Figure 4 shows the condensed FE model of the GLR configuration including markers (triangles) for 


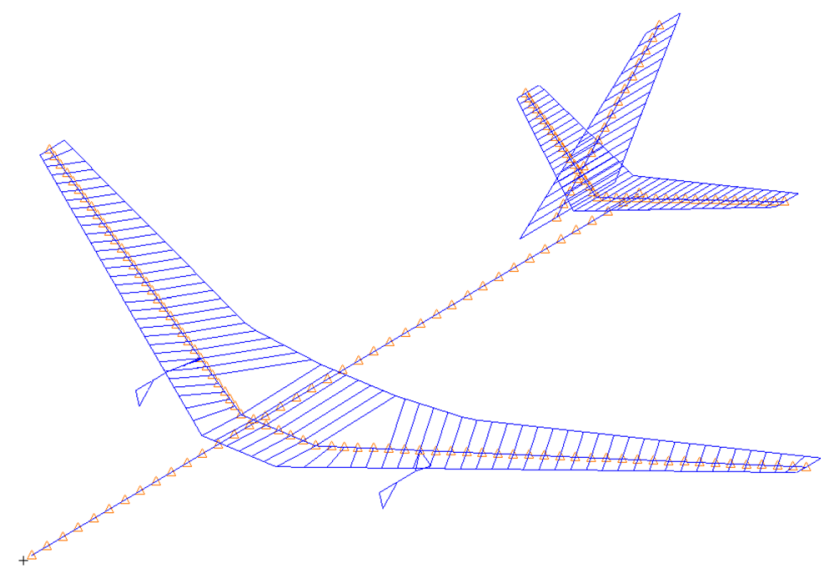

Fig. 4 Condensed FE model of the GLR configuration with markers for the load reference axis nodes

the LRA nodes. Subsequently, the process conducts 1-cos design gust, $1 \mathrm{~g}$ trim and manoeuvre simulations. In the simulations, no engine thrust is considered. Since the aerodynamic modelling of MSC.Nastran is based on the potential theory, aerodynamic nonlinearities such as transonic effects are not taken into account. Nevertheless, DLM corrections using CFD data, e.g. for gust encounters as shown by Kaiser et al. [4] and Quero [5], can be considered. On the other hand, such implementation increases the computing time. Since the loads from the 1-cos design gust simulations are only incremental loads, they have to be superposed with the $1 \mathrm{~g}$ trim loads to obtain the total loads. This step is followed by filtering the loads that are relevant for the structural optimization using 2D load envelopes, see Ref. [6], and the component-wise structural optimization itself. In doing so, the objective is the minimization of the wing box or empennage box mass respectively, while complying with strain and buckling constraints. Moreover, an additional optimization with aeroelastic constraints-in this case aileron effectiveness-is conducted. If an aileron reversal occurs below $V_{\mathrm{D}}+15 \%$ as defined in CS-25 [7], the wing is re-optimized, and only an increase in the material thicknesses is allowed, see Ref. [8]. The cycle with the loads analysis and structural optimization is repeated until the relative change of the structural mass between two cycles is below $0.5 \%$. After the masses have converged, a subsonic flutter check of the passive aircraft is run to ensure that the aircraft does not flutter in the prescribed flight envelope.

For the design process and for the ride comfort investigation in this work, the reference aircraft is equipped with load alleviation functions comprising manoeuvre load alleviation (MLA) and GLA based on Ref. [6]. The MLA and GLA is designed to reduce the wing bending moment during manoeuvres and discrete gust encounters which are simulated according to CS-25 [7] respectively. The load

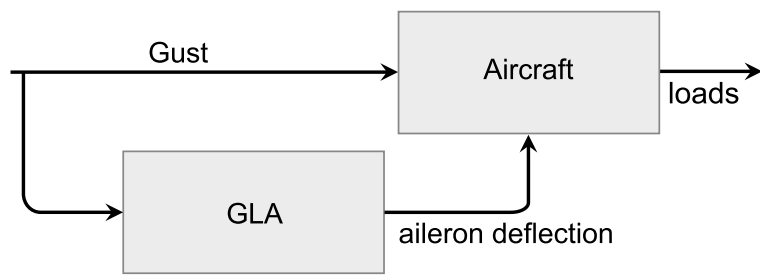

Fig. 5 Block diagram of feed-forward GLA

alleviation leads to a reduction of the structural mass on the wing. To quantify the effect of load alleviation on the structural mass of the particular reference aircraft, however, an additional load analysis and structural optimization run is necessary and it is not in the scope of this work. As a reference nevertheless: MLA and GLA can achieve a mass reduction between $0.3 \%$ and $0.9 \%$ of OEM as Handojo [9] shows for mid-range aircraft. The MLA and GLA are active at the same time in case a manoeuvre and a gust encounter occurs at the same time. Since the focus of this work lies in the passenger ride comfort during cruise in turbulent air, the MLA has zero output since no manoeuvre is commanded. Therefore, only the GLA is explained in detail.

\subsection{Gust load alleviation}

The GLA employs a feed forward control algorithm with signals from the angle of attack sensors as input and symmetric aileron deflection as output. Figure 5 illustrates the signal flow.

The angle of attack sensors are assumed to be placed at the aircraft nose. The input parameter for the GLA is the angle of attack increment due to gust $\alpha_{\mathrm{g}}$ based on König et al. [10] as defined by

$\alpha_{\mathrm{g}}=\alpha_{\mathrm{m}}-\Theta+\frac{\dot{z}}{V_{\mathrm{TAS}}}+\frac{q \cdot\left|r_{\mathrm{CG}}\right|}{V_{\mathrm{TAS}}}+\Delta \alpha_{\mathrm{str}, \mathrm{corr}} \approx \frac{w_{\mathrm{wg}}}{V_{\mathrm{TAS}}}$,

with the measured angle of attack $\alpha_{\mathrm{m}}$, pitch angle $\Theta$, vertical speed $\dot{z}$, true airspeed $V_{\mathrm{TAS}}$, pitch rate $q$, distance between aerodynamic sensor and aircraft centre of gravity $r_{\mathrm{CG}}$, correction term for the structural oscillations in the case of a flexible aircraft $\Delta \alpha_{\text {str,corr }}$ and the vertical wind speed $w_{\text {wg }}$. In the simulation model, an ideal measurement of the angle of attack is assumed.

The GLA commands symmetric ailerons since it is effective in reducing the bending moment increment due to gusts. A deflection of the outer spoilers can be considered as well; however, spoiler deflections increase drag and evoke longitudinal accelerations which are undesirable. Moreover, elevator deflections are not taken into account either since they are ineffective at frequencies 
around the first wing bending mode, at which the highest gust loads typically occur at the wing.

For the GLA transfer function, the symmetric aileron deflection is set to be proportional to the gust angle of attack, and the GLA is mainly synthesized to reduce loads during 1-cos gust encounters according to CS-25 [7]. Since discrete gust encounters are transient phenomena and the maximum loads are reached within a second after the gust is detected at the aircraft nose, an integral term is not necessary. A second-order low pass filter for the GLA is set at $10 \mathrm{~Hz}$ to avoid excessive aileron deflection rates. Moreover, to avoid having aileron deflection in a constant vertical wind field, a second-order high-pass filter is set at $0.1 \mathrm{~Hz}$.

Concerning the delay time, the flight control computer (FCC) is assumed to process the angle of attack signal measured at the nose and output the aileron deflection command when the gust arrives at the wing. The actuator model includes limiters for deflection rate $\left(\dot{\xi}_{\max }=40\right.$ $\mathrm{deg} / \mathrm{s})$ and amplitude $\left(\xi_{\max }=10 \mathrm{deg}\right)$. The bandwidth of the actuator is assumed to be covered by the low-pass filter of the GLA already. The GLA transfer function $\operatorname{GLA}(s)$ is defined in the frequency domain by

$\mathrm{GLA}(s)=\frac{\xi_{\mathrm{c}}(s)}{\alpha_{\mathrm{g}}(s)}=k_{\mathrm{GLA}} \cdot \mathrm{LP}(s) \cdot \mathrm{HP}(s) \cdot \mathrm{TD}(s)$,

with the commanded symmetrical aileron deflection $\xi_{\mathrm{c}}$, the proportional control gain $k_{\mathrm{GLA}}=-2.0$, low-pass filter transfer function $\operatorname{LP}(s)$, high-pass filter function $\operatorname{HP}(s)$ and time delay transfer function $\mathrm{TD}(s)$ where

$\operatorname{LP}(s)=\left(\frac{1}{\frac{s}{2 \pi f_{\mathrm{LP}}}+1}\right)^{2}$,

with the low-pass cutoff frequency $f_{\mathrm{LP}}=10 \mathrm{~Hz}$,

$\mathrm{HP}(s)=\left(\frac{\frac{s}{2 \pi f_{\mathrm{HP}}}}{\frac{s}{2 \pi f_{\mathrm{HP}}}+1}\right)^{2}$,

with the high-pass onset frequency $f_{\mathrm{HP}}=0.1 \mathrm{~Hz}$,

$\operatorname{TD}(s)=\mathrm{e}^{-2 \pi \cdot i f \cdot t_{\text {del }}} \quad$ and $\quad t_{\text {del }}=f\left(V_{\mathrm{TAS}}\right)$.

The commanded aileron deflection $\xi_{\mathrm{c}}$ is then limited in the deflection and its rate. Hence, the actual aileron deflection $\xi$ becomes

$\xi(t)=\xi_{\mathrm{c}}(t) \cdot f_{\mathrm{lim}}(t)$.

The GLA, that is designed to reduce loads during discrete gust encounters, is also used for the analyses with continuous turbulence.

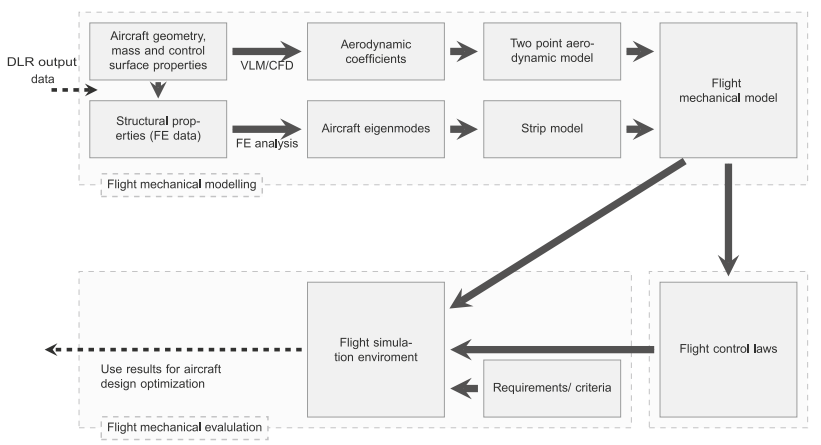

Fig. 6 MITRA: signal flow diagram for modelling and evaluating a flexible aircraft

\subsection{Export for flight mechanical evaluation}

For the flight mechanical evaluation, the aeroelastic model as well as the GLA algorithm are exported to MITRA. The transferred data of the aircraft model consists of the load reference axis (LRA) grid positions, the mode frequencies as well as the respective mode shapes of nine mass configurations ranging from the operating empty mass (OEM) to the maximum take-off mass (MTOM).

\section{Flight mechanical evaluation}

TUB developed Multi-objective Evaluation of Preliminary Aircraft Designs (MITRA) for flight mechanical evaluation of preliminary aircraft designs. MITRA automatically generates a flight mechanical model of the flexible aircraft by using the nonlinear six-degree-of-freedom equations of the rigid-body motion plus the structural input data described in Sect. 3.3 and supplements it with FCL including GLA. With those elements, MITRA conducts flight simulation to mimic flight tests and uses the results to perform flight mechanical evaluations. MITRA uses design and certification requirements (from Refs. [7, 11, 12]) to define the virtual flight tasks for all relevant critical test points (a combination of weight, centre of gravity, initial conditions, etc.) and to derive flight mechanical assessment criteria.

Figure 6 shows all process steps from aircraft modelling to the evaluation. Each element has been described in detail and published: the flight mechanical modelling of a flexible aircraft in Ref. [13], the automatic generation of the FCL in Ref. [14] and the flight simulation test environment in Refs. [3, 15].

The modelling of the flexible aircraft consists of two steps: the building of the aerodynamic model for the rigid aircraft dynamics and a model to include aeroelastic influences. The aerodynamic model calculates the forces and moments that the airflow induces on the rigid aircraft. The 


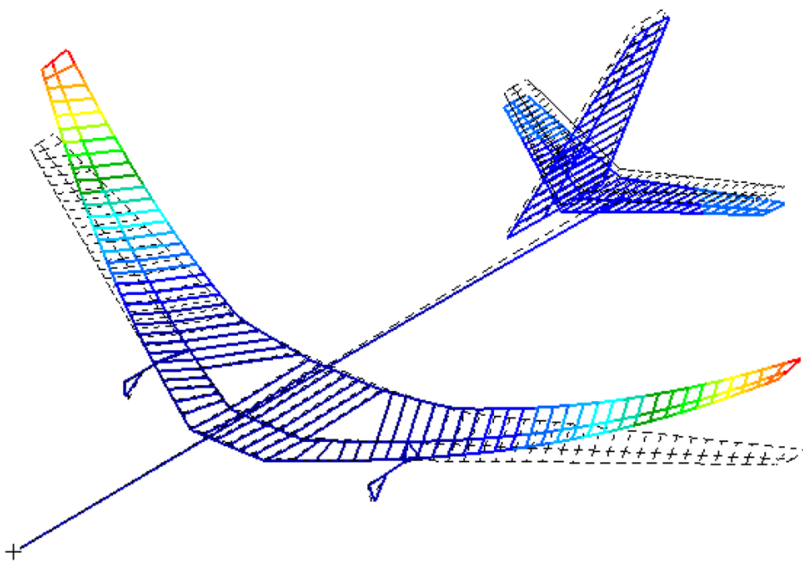

Fig. 7 First symmetric wing bending mode of the GLR configuration

model requires for this task look-up tables with aerodynamic coefficients and derivatives, for which MITRA uses the vortex-lattice-method (VLM). MITRA connects the trailing edge and leading edge of each lifting surface in the threedimensional space and partitions it spanwise and chordwise into large panels (with wing profiles) for VLM calculations at different states. Aerodynamic properties of non-lifting surfaces are calculated using semi-empirical methods from Ref. [16]. MITRA allows importing aerodynamic data from high-fidelity CFD methods for more accurate flight mechanical models.

To consider the impact of aeroelasticity, finite element (FE) methods combined with an aerodynamic strip model calculate the elastic deformations under loads. The MONA process calculates the eigenvalues and corresponding eigenvectors (eigenforms) of the aircraft structure. The eigenvalues contain the frequency of each eigenform, while the eigenvectors contain the translational and angular displacements. Figure 7 shows exemplarily the first symmetric bending eigenform of the reference aircraft.

MITRA builds a strip model to calculate the forces and moments that are depending on the translational, rotational deformation and on the control surface deflection of each strip of the flexible structure. The strip theory is a simplified method that discards 3D effects in the lift generation. These effects are mainly produced at the wing tip and are especially important for low aspect-ratio wings. For aircraft with high aspect-ratio wings, which is the case for the current study, the strip theory provides a good approximation, with the advantage of an easy numerical implementation for flexible aircraft dynamics in the time domain, compared to an approximation of the doublet-lattice-method (DLM) for the time domain. The motion of each strip is described by the structural dynamic differential equation using the modal superposition method, which do not include rigidbody modes,

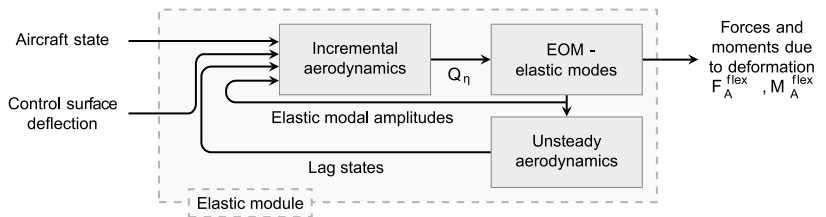

Fig. 8 Elastic module of the flight mechanical model

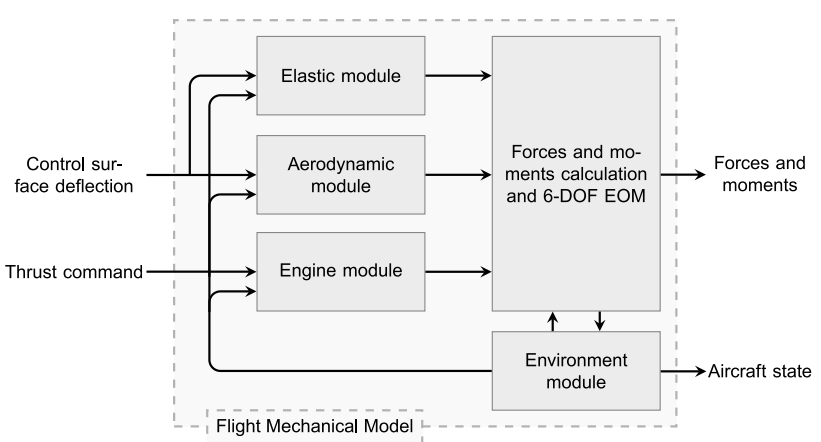

Fig. 9 Flight mechanical model

$\underline{\underline{\mu}} \underline{\underline{\eta}}(t)+\underline{\underline{\beta}} \underline{\dot{\eta}}(t)+\underline{\gamma} \underline{\underline{\eta}}(t)=\underline{Q}(t)$,

with modal amplitude $\eta$, the modal mass $\underline{\mu}$, the damping $\underline{\beta}$, the stiffness matrix $\underline{\gamma}$ and the generalized force $\underline{Q}$.

This method composes the overall motion of the structure from the contributions of each structural eigenform. Due to the low influence of high-frequency structural eigenforms on the flight dynamical behaviour, modes with a frequency over $30 \mathrm{~Hz}$ are neglected. Geometrical properties of the strips derive from grid points of the FE-model. Unsteady aerodynamic effects are implemented by Duhamel integral and an exponential approximation of the Wagner function as described in Ref. [17]. MITRA uses an elastic module for the implementation, as shown in Figs. 8 and 9).

The modelling process integrates the aerodynamic and strip model intro modules that are part of the flight mechanical model, see Fig. 9. Other implemented modules are the environmental module that computes the wind conditions, gravitational acceleration, Mach number and air density as a function of flight altitude and the engine module that outputs thrust forces and moments. The flight mechanical model uses control surface deflections and thrust commands as inputs to calculate the resulting forces and moments acting on the aircraft. They are the inputs to solve the six-degreeof-freedom equations of motion and to calculate the resulting accelerations. The numerical integration method for the acceleration is the second-order Runge-Kutta method. This process is repeated for each time step of $1 \mathrm{~ms}$ to consider elastic frequencies. 
Table 3 Handling qualities requirements

\begin{tabular}{ll}
\hline Handling quality & Requirement from MIL-STD-1797A \\
\hline Short period oscillation & $0.3>D>2 ; \omega_{0}>0.7 \frac{\mathrm{rad}}{\mathrm{s}}$ \\
Phugoid oscillation & $D>0.04$ \\
Dutch roll oscillation & $\omega_{0}>0.4 \frac{\mathrm{rad}}{\mathrm{s}} ; D>0.08 ; D \cdot \omega_{0}>0.15$ \\
Roll motion & $0.3 \mathrm{~s}<T_{\mathrm{R}}<1.4 \mathrm{~s}$ \\
Spiral oscillation & $T_{2}>20 \mathrm{~s}$ \\
\hline
\end{tabular}

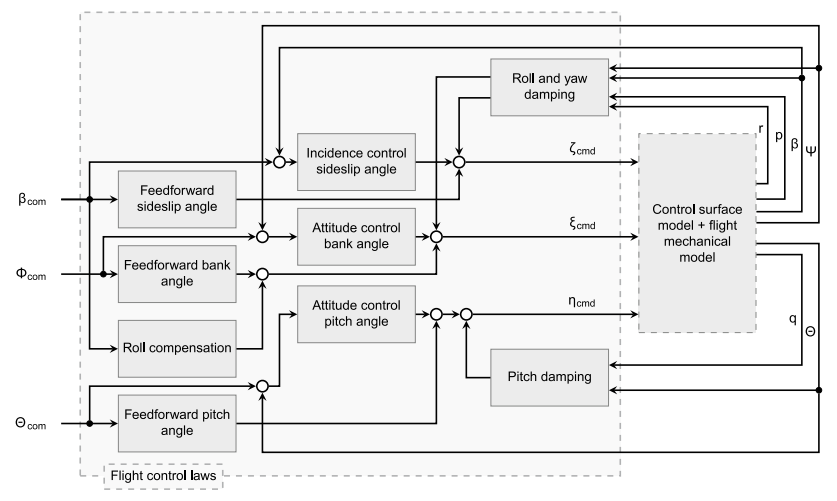

Fig. 10 Implemented FCL

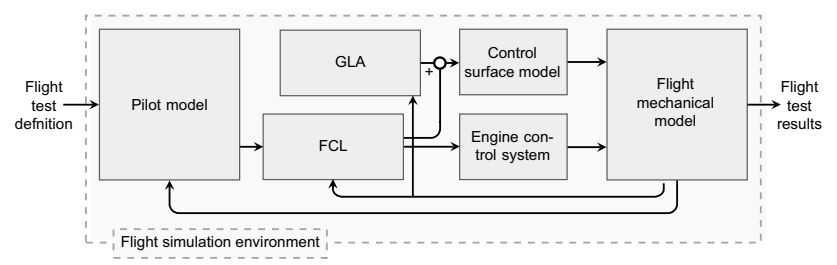

Fig. 11 MITRA simulation environment

All modern transport aircraft use FCL to improve handling qualities, for protection and to provide consistent behaviour within the flight envelope and must be considered for the assessment. MITRA uses a predefined preliminary flight control architecture and functions shown in Fig. 10 to automatically generate common FCL and links them to the flight mechanical model. The FCL provide attitude control and consist of inner and outer loops. The inner loops include pitch, roll and yaw damping, which improves and unifies handling qualities across the flight envelope according to criteria from MIL-STD-1797A [12] shown in Table 3.

The outer loop consists of feedforward and feedback controllers for aircraft attitude and incidence. In an automated manner, the design process derives the control parameters from linearized flight mechanical models and time-domain optimization. The GLA functions (described in Sect. 3.2) output aileron commands, which are added to the ones from the FCL, as shown in Fig. 11 and as described in Ref. [18].
Thrust control is not considered in this study. The bandwidth of thrust control is expected to be so low $(\ll 1 \mathrm{~Hz})$ that it is not relevant for the ride comfort according to Fig. 14.

MITRA integrates the flight mechanical model and the FCL into a flight simulation test environment as shown in Fig. 11. This environment simulates criteria-based flight tests and evaluates its results in an automated manner for all eligible flight envelope points. In the simulations, a pilot model computes control commands to perform the flight task. In a feedback loop, the flight mechanical model provides data for the pilot model and FCL until the flight task is completed. TUB originally developed MITRA for criteria derived from civilian and military certification specifications, but additional assessments have been included. MITRA's modular design allows the exchange of all modules.

\section{Passenger ride comfort criteria}

Passenger ride comfort during cruise flight has a significant impact on the personal experience of a flight. Multiple factors influence ride comfort, including accelerations acting on the passengers (vibrations), acoustic, available space, flight duration, for which accurate models are needed. From a flight mechanical perspective, the most important (and only) contribution to comfort are the accelerations acting on the passengers. The perceived comfort relates to the amplitude, direction, frequency and duration of the accelerations. Here, only the influence of accelerations on passengers ride comfort is considered.

Structural vibrations can be caused (but not only) either by turbulence or by gust encounters. In cruise flight at high altitudes, atmospheric turbulence is the dominant contributor to discomfort. It is important to reduce accelerations acting on passengers for a comfortable flight as much as possible. This work investigates vibrations with frequencies up to $25 \mathrm{~Hz}$. For the given turbulence spectrum, the energy content above $25 \mathrm{~Hz}$ is negligible, see bottom plot in Fig. 17. A total of 50 elastic modes are taken into account.

Certification specifications do not provide requirements for passenger ride comfort. For a flight mechanical investigation, NASA developed a general empirical model for the prediction of passenger discomfort in the presence of vibration (and noise) using the root mean square (RMS) of the acting accelerations on passengers as the input parameter. NASA conducted experiments with approximately 2200 test subjects to gather empirical data for the model, as described in detail in Ref. [19]. A simplified model was developed in Ref. [20], that provides satisfactory assessments. This ride comfort model is used here. RMS is defined as the arithmetic mean of the squares of a set of $n$ values $x_{1}, x_{2}, \ldots, x_{n}$, 


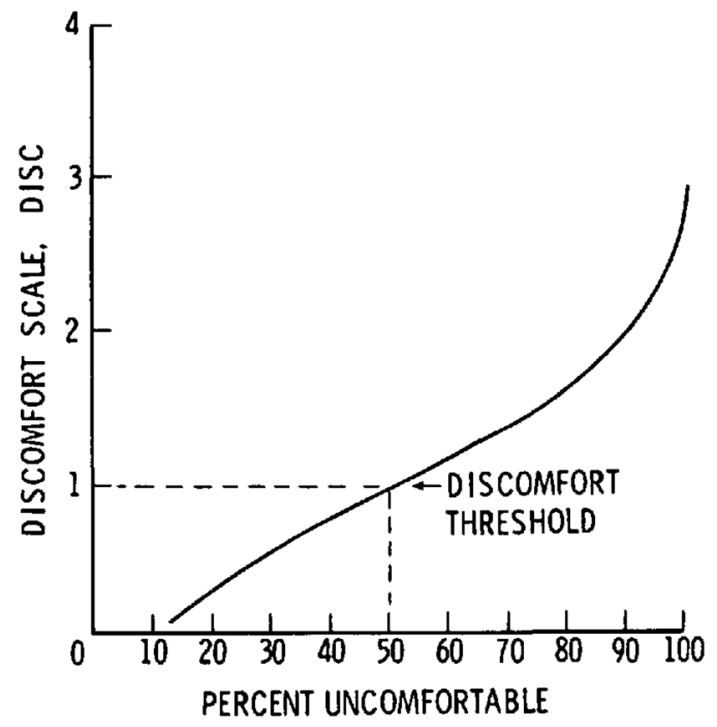

Fig. 12 Discomfort or DISC as a function of percentage of passengers that feel uncomfortable, from Ref. [19]

$x_{\mathrm{RMS}}=\sqrt{\frac{1}{n}\left(x_{1}^{2}+x_{2}^{2}+\cdots+x_{n}^{2}\right)}$.

Obviously, lower RMS values of disturbing accelerations are desirable. The range of vibration (and acoustic noise) used to derive the model includes amplitudes and frequencies that are known to impact passenger comfort. The model accounts for the effects of combined axis vibrations (up to three axes simultaneously).

For every source of discomfort, NASA developed a formula for $D_{L_{-}}$values (where ${ }_{-}{ }_{-}$is a placeholder for a discomfort contribution) based on the conducted survey. Reference [19] defines the discomfort factor as a single numerical descriptor of passenger discomfort which is measured along a ratio scale of discomfort that is anchored at discomfort threshold. Figure 12 shows the relationship between the discomfort scale (ordinate) and the corresponding percentage (abscissa) of passengers who would rate that discomfort level as being uncomfortable. A value of 1.0 on the discomfort scale represents to the discomfort threshold, i.e., $50 \%$ of the passengers would be uncomfortable. These units are measured along a ratio scale so that all estimated values bear a direct ratio relationship to one another. For example, $D_{\text {vert }}=2$, means that around $90 \%$ of the passengers would find the vertical accelerations uncomfortable, while at $D_{\text {vert }}=0.5$, only about $30 \%$ would find those accelerations uncomfortable.

The $D$ values of the respective sources of discomfort are then weighted and superposed to obtain the total discomfort value due to vibration $D_{\mathrm{VIB}}$. Figure 13 shows the signal flow of the passenger discomfort model. The NASA model allows measuring the vibrations at any seat location. Although the

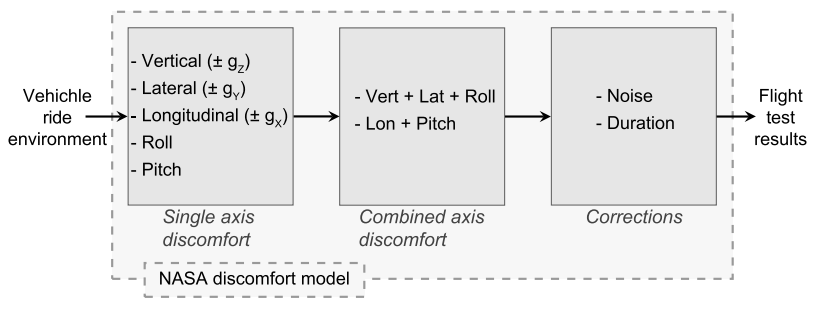

Fig. 13 NASA passenger discomfort model

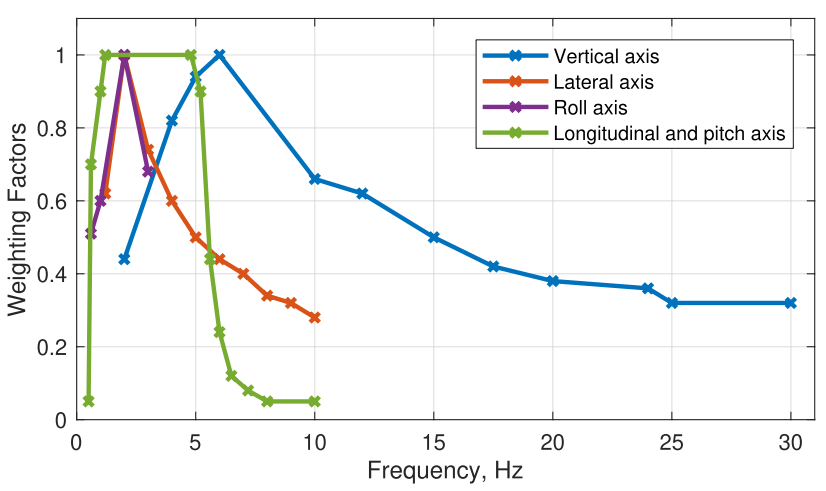

Fig. 14 Frequency weight plot for all axes, see Ref. [20]

cockpit and the most rear seats will have more accelerations than seats at the c.g. position, this work only considers the acceleration at the c.g. position. The structural model in MITRA only allows extracting the acceleration at c.g. Additional acceleration measurement points will be added in future work.

The calculation of the different discomfort factors requires flight simulations at relevant test points. The starting conditions are trimmed unaccelerated straight and level flights with enabled FCL (normal law-NL) and GLA off or on. The simulation duration is $1000 \mathrm{~s}$. During the simulation, the pilot model does not command any inputs to control the aircraft. The attitude flight control law maintains aircraft attitude.

The aircraft is excited by wind turbulence in all axis directions. The turbulence is modelled with the Dryden model as it allows simulation in the time domain.

MITRA records all relevant flight states after each simulation. The ride comfort model requires the accelerations (first column in Table 4) as input data. The vibrations are measured at the c.g., They are transformed from the time domain into the frequency domain using the Fourier transformation. The resulting power spectrum density (PSD) of each acceleration fluctuation is weighted depending on the frequency weighting factors from Fig. 14.

The four frequency weightings reflect human comfort sensitivity to vibration frequencies for that particular axis. 
Table 4 RMS calculation for each relevant direction

\begin{tabular}{ll}
\hline Input acceleration & Weighted RMS \\
\hline$a_{\mathrm{x}}$, in $\mathrm{m} / \mathrm{s}^{2}$ & $\left(g_{\mathrm{w}}\right)_{\text {lon }}$, in $\mathrm{g}\left(9.81 \mathrm{~m} / \mathrm{s}^{2}\right)$ \\
$a_{\mathrm{y}}$, in $\mathrm{m} / \mathrm{s}^{2}$ & $\left(g_{\mathrm{w}}\right)_{\text {lat }}$, in $\mathrm{g}\left(9.81 \mathrm{~m} / \mathrm{s}^{2}\right)$ \\
$a_{\mathrm{z}}$, in $\mathrm{m} / \mathrm{s}^{2}$ & $\left(g_{\mathrm{w}}\right)_{\text {vert }}$, in $\mathrm{g}\left(9.81 \mathrm{~m} / \mathrm{s}^{2}\right)$ \\
$\ddot{\Phi}$, in $\mathrm{rad} / \mathrm{s}^{2}$ & $\ddot{\Phi}_{\mathrm{w}}$, in rad$/ \mathrm{s}^{2}$ \\
$\ddot{\Theta}$, in $\mathrm{rad} / \mathrm{s}^{2}$ & $\ddot{\Theta}_{\mathrm{w}}$, in $\mathrm{rad} / \mathrm{s}^{2}$ \\
\hline
\end{tabular}

The RMS acceleration level of each weighted spectrum was then determined and are inputs for the following steps.

Equations 9-13 calculate the discomfort factors due to vertical acceleration $D_{\text {vert }}$, lateral acceleration $D_{\text {lat }}$, longitudinal acceleration $D_{\text {long }}$, angular roll acceleration $D_{\text {roll }}$ and angular pitch acceleration $D_{\text {pitch }}$ in their respective (right hand) axes. The weighted RMS values, for which the translational accelerations are normalized for earth gravity acceleration $g$ (second column in Table 4) are input values.

$$
\begin{aligned}
& D_{\text {vert }}= \begin{cases}0.241+44.672\left(g_{\mathrm{w}}\right)_{\text {vert }}, & \text { if }\left(g_{\mathrm{w}}\right)_{\text {vert }}>0.01 \mathrm{~g} \\
68.772\left(g_{\mathrm{w}}\right)_{\text {vert }}, & \text { otherwise },\end{cases} \\
& D_{\text {lat }}= \begin{cases}0.393+47.494\left(g_{\mathrm{w}}\right)_{\text {lat }}, & \text { if }\left(g_{\mathrm{w}}\right)_{\text {lat }}>0.01 \mathrm{~g} \\
86.794\left(g_{\mathrm{w}}\right)_{\text {lat }}, & \text { otherwise },\end{cases} \\
& D_{\text {long }}=-0.02+42.24\left(g_{\mathrm{w}}\right)_{\text {long }}, \\
& D_{\text {roll }}= \begin{cases}-0.21+4.506 \ddot{\Phi}_{\mathrm{w}}, & \text { if } \ddot{\Phi}_{\mathrm{w}} \geq 0.10 \frac{\mathrm{rad}}{\mathrm{s}^{2}} \\
2.406 \ddot{\Phi}_{\mathrm{w}}, & \text { otherwise and }\end{cases} \\
& D_{\text {pitch }}= \begin{cases}0.41+5.07 \ddot{\Theta}_{\mathrm{w}}, & \text { if } \ddot{\Theta}_{\mathrm{w}} \geq 0.116 \frac{\mathrm{rad}}{\mathrm{s}^{2}} \\
8.62 \ddot{\Theta}_{\mathrm{w}}, & \text { otherwise. }\end{cases}
\end{aligned}
$$

The results from Eqs. 9 to 13 are used to calculate the combined axis discomfort factor for vertical, lateral and roll vibrations $D_{\mathrm{VLR}}$. For this, $D_{\text {vert }}, D_{\text {lat }}$ and $D_{\text {roll }}$ are ranked by the value and stored (highest to lowest) in $D_{1}, D_{2}$ and $D_{3}$. The values are inputs to calculate the temporary discomfort factors $D_{4}, D_{\mathrm{c} 1}$ and $D_{\text {comb1 }}$.

$D_{4}=\sqrt{D_{2}^{2}+D_{3}^{2}}$

$D_{\mathrm{c} 1}=\sqrt{D_{1}^{2}+D_{2}^{2}+D_{3}^{2}}$

$D_{\text {comb } 1}= \begin{cases}-0.44+1.65 D_{\mathrm{c} 1}, & \text { if } D_{\mathrm{c} 1} \geq 0.88 \\ 1.14 D_{\mathrm{c} 1}, & \text { otherwise }\end{cases}$

$D_{\mathrm{VLR}}$ is then calculated by
$D_{\mathrm{VLR}}= \begin{cases}D_{1}+\frac{D_{4}\left(D_{\text {comb } 1}-D_{1}\right)}{0.4}, & \text { if } D_{4}<0.4 \cap \frac{D_{1}}{D_{2}} \geq 3 \\ D_{\text {comb } 1}, & \text { otherwise. }\end{cases}$

The discomfort factors $D_{\text {pitch }}$ and $D_{\text {long }}$ are ranked by the value and stored (highest to lowest) in $D_{5}$ and $D_{6}$. The values are inputs to calculate the temporary discomfort factors $D_{\mathrm{c} 2}$

$D_{\mathrm{c} 2}=\sqrt{D_{5}^{2}+D_{6}^{2}}$

and $D_{\text {comb2 }}$

$D_{\text {comb2 }}= \begin{cases}-1.07+1.77 D_{\mathrm{C} 2}, & \text { if } D_{\mathrm{c} 2} \geq 1 \\ 0.7 D_{\mathrm{c} 2}, & \text { otherwise and }\end{cases}$

The combined axis discomfort due to longitudinal and pitch vibration $D_{\mathrm{LP}}$ becomes

$D_{\mathrm{LP}}= \begin{cases}D_{5}+\frac{D_{6}\left(D_{\mathrm{comb} 2}-D_{5}\right)}{0.4}, & \text { if } D_{6}<0.4 \cap \frac{D_{5}}{D_{6}} \geq 3 \\ D_{\text {comb2 } 2}, & \text { otherwise. }\end{cases}$

Finally, the combined discomfort for vibration $D_{\mathrm{VIB}}$ (in this work not corrected for noise and duration) is

$D_{\mathrm{VIB}}=\sqrt{D_{\mathrm{VLR}}^{2}+D_{\mathrm{LP}}^{2}}$.

\section{Demonstration of the method}

\subsection{Test description}

MITRA applies the ride comfort model to the reference aircraft described in Sect. 2. The DLR load analysis and structural design process provides the necessary input data for MITRA to build a flight simulation model.

Then, test cases for the evaluation are defined in MITRA. Ride quality in turbulent flight is investigated during cruise condition, which makes up a large fraction of the total flight time. For the initial condition, a cruise altitude of $11,000 \mathrm{~m}$ and cruise Mach number of 0.82 is selected. The FCL are in attitude hold control mode, which is representative for automatic flight. ${ }^{1}$ Two load conditions are selected to be investigated: the operating empty mass (OEM) and the maximum take-off mass (MTOM). They are combined with the most forward c.g. (FWD) and most rear c.g.(AFT) position. Each case is tested with GLA off and on. Table 5 shows the resulting test case list with its varying parameter. Our results refer to the lightest (OEM) and heaviest (MTOM) mass

\footnotetext{
1 Although MITRA can investigate FCS failures, they are not considered a main driver for ride comfort investigations, since safety is more important in those cases.
} 
Table 5 All tests are conducted at an altitude of 11,000 $\mathrm{m}$ and Mach number of 0.82 and in attitude hold mode

\begin{tabular}{llll}
\hline Test case & Mass $(\mathrm{kg})$ & CG & GLA \\
\hline 1 & 122,000 & FWD & OFF/ON \\
2 & 122,000 & AFT & OFF/ON \\
3 & 235,000 & FWD & OFF/ON \\
4 & 235,000 & AFT & OFF/ON \\
\hline
\end{tabular}

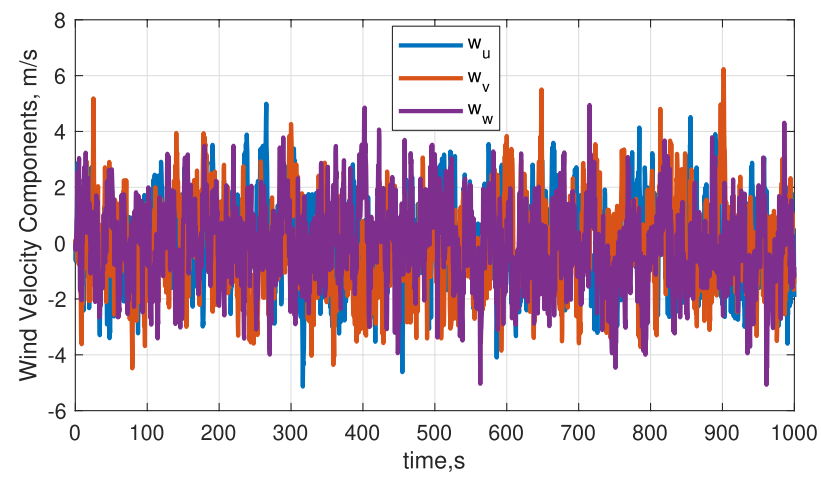

Fig. 15 Wind turbulence for all three axes

configurations of the reference aircraft, and further mass configurations between OEM and MTOM are expected to yield results that lie in between. MITRA simulates each test case with turbulence excitation in all directions (as shown in Fig. 15) for a duration of $1000 \mathrm{~s}$.

\subsection{Simulation results}

Figure 16 shows simulation results for Test Case 1 (which is used exemplary for the other test cases) with GLA disabled (off) in the time-domain. The top plot shows the aircraft translational acceleration in all three directions and the bottom plot shows the angular acceleration in roll and pitch direction. By comparing the translational motion, it can be seen that the aircraft vertical acceleration is significantly higher than the ones in longitudinal and lateral direction. This is because the winds acts on the available aircraft surface areas, for which the one in vertical direction is much higher. Concerning angular accelerations, the pitch and roll acceleration have a similar magnitude.

Table 6 shows the unweighted and weighted RMS values for each acceleration. As it can be already seen in Fig. 16, the unweighted RMS value for the vertical acceleration $a_{z}$ is much higher than the other ones. The same can be said for the weighted RMS. It can be also seen that the weighted RMS for longitudinal acceleration $a_{x}$ is
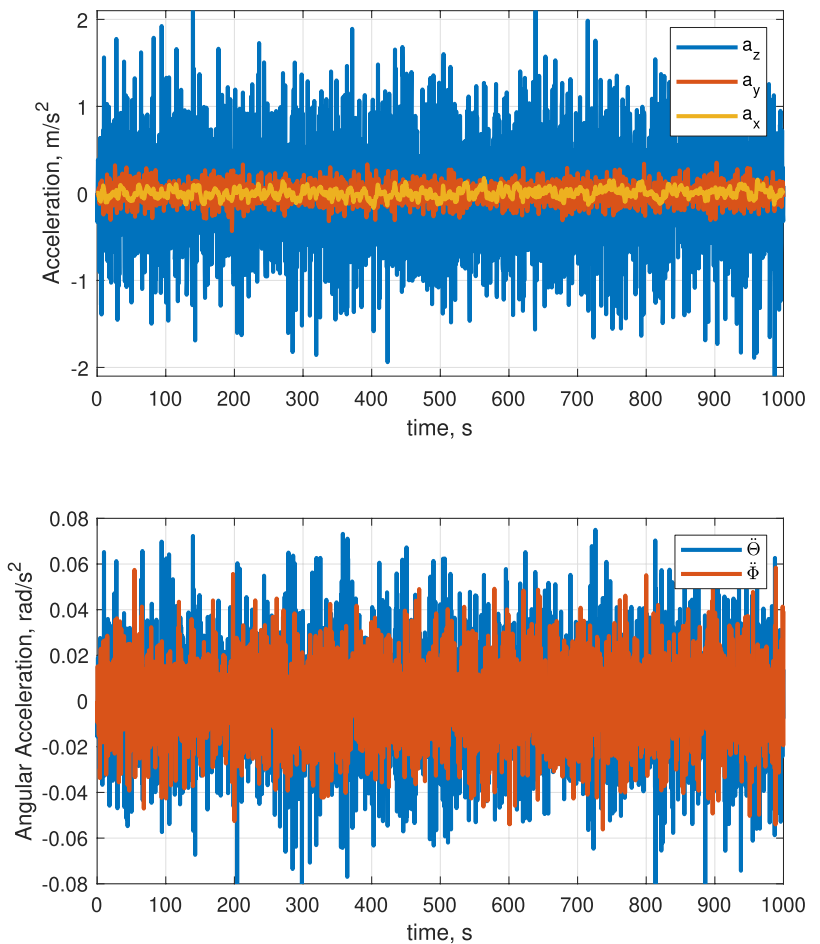

Fig. 16 Time response of Test Case 1 with GLA off

Table 6 RMS values for all simulations

\begin{tabular}{llll}
\hline Acc. & GLA & RMS unweighted & RMS weighted \\
\hline$a_{x}$ & OFF/ & 0.05 & 0.00 \\
& ON & 0.05 & 0.00 \\
$a_{y}$ & OFF/ & 0.11 & 0.05 \\
& ON & 0.11 & 0.05 \\
$a_{z}$ & OFF/ & 0.64 & 0.31 \\
& ON & 0.60 & 0.29 \\
$\ddot{\Theta}$ & OFF/ & 0.02 & 0.01 \\
& ON & 0.02 & 0.01 \\
$\ddot{\Phi}$ & OFF/ & 0.02 & 0.02 \\
& ON & 0.02 & 0.02 \\
\hline
\end{tabular}

zero, meaning that they will not impact discomfort at all. Table 6 also shows that with GLA enabled (GLA ON), the RMS value for $a_{\mathrm{z}}$ is reduced, while the other RMS values are unaffected.

Figure 17 shows in the top plot the PSD of the applied Dryden turbulence with a scale of turbulence of $2500 \mathrm{ft}$ $(762 \mathrm{~m})$ in the frequency domain. The turbulence scale $L_{\text {turb }}$ is set to $(762 \mathrm{~m}) 2500 \mathrm{ft}$ to match the value given in CS25.341(b)(2) [7]; the Dryden turbulence spectrum for the vertical wind speed is defined by 

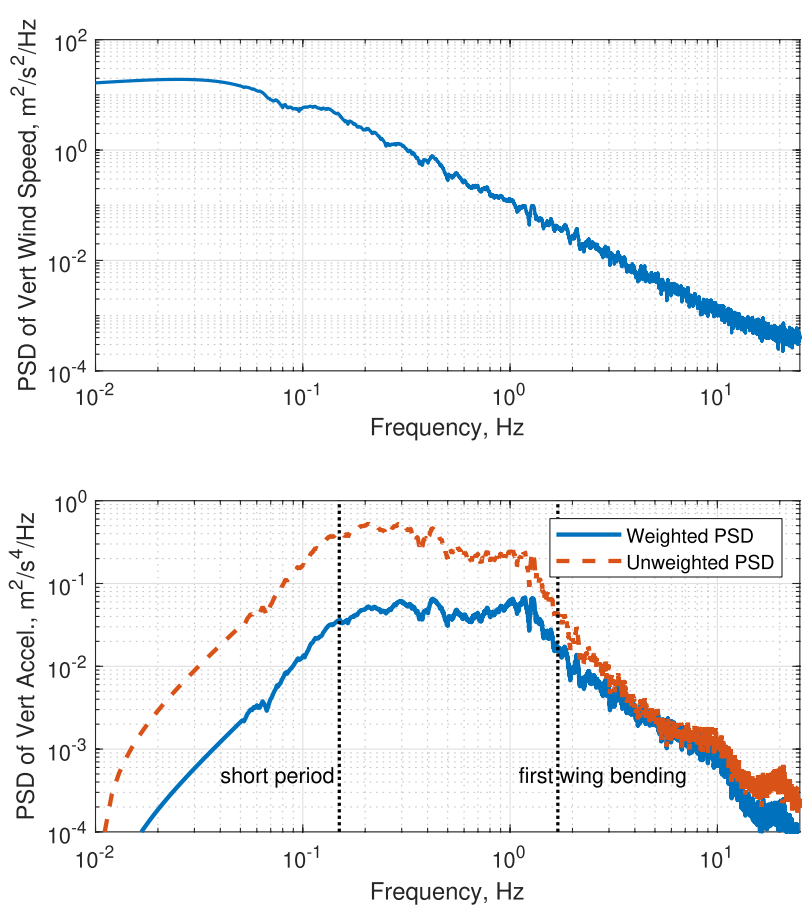

Fig. 17 Power spectral density of the vertical wind speed (top) and of the the vertical acceleration response to turbulence (bottom)

$\operatorname{PSD}_{\mathrm{wg}}(f)=\mathrm{rms}_{\mathrm{wg}}^{2} \frac{2 L_{\mathrm{turb}}}{V_{\mathrm{TAS}}} \cdot \frac{1+3\left(L_{\mathrm{turb}} \frac{2 \pi f}{V_{\mathrm{TAS}}}\right)^{2}}{\left[1+\left(L_{\mathrm{turb}} \frac{2 \pi f}{V_{\mathrm{TAS}}}\right)^{2}\right]^{2}}$,

with the power spectral density of the vertical wind speed $\mathrm{PSD}_{\mathrm{wg}}$, RMS of the vertical wind speed $\mathrm{rms}_{\mathrm{wg}}$, scale of turbulence $L_{\text {turb }}$ and true airspeed $V_{\text {TAS. }}$. In this case, the Dryden spectrum is selected since it has integer exponents, so that the spectrum can be transformed into the time domain analytically, since the result evaluation is conducted in the time domain. The RMS of the wind speed represents moderate turbulence. It is defined for a probability of exceedance of $10^{-3} 1 / \mathrm{h}$ according to MIL-F-8785C [11]. This yields an RMS of each wind component of approx. $1.37 \mathrm{~m} / \mathrm{s}(4.5 \mathrm{ft} / \mathrm{s})$ TAS for the reference cruise condition.
For Test Case 1 Fig. 17 shows in the bottom plot the PSD of the vertical acceleration at the aircraft c.g. as a response to the turbulence. The frequencies of the short period mode and the first wing bending mode are marked. The term unweighted PSD refers to the actual response of the aircraft, whereas the weighted PSD, which is weighted according to Fig. 14, is the input for the ride comfort investigation.

As a remark: with a simulated time of $1000 \mathrm{~s}$ and a time increment of $20 \mathrm{~ms}$, the maximum observable frequency according to the Nyquist-Shannon theorem (see Ref. [21]) is $25 \mathrm{~Hz}$, and the frequency increment is $0.001 \mathrm{~Hz}$. To prevent having a large noise band in the visualization in the frequency domain, a smoothing Savitzky-Golay filter (see Ref. [22]) with a polynomial order of 3 and a frame size of 151 is applied.

\subsection{Discomfort results}

Table 7 shows discomfort values of each single axis/direction for all test cases with GLA enabled (on) and disabled (off). $D_{\text {vert }}$ has a higher magnitude than the other discomfort values in all test cases and therefore is the dominant singleaxis impact factor for discomfort. $D_{\text {lat }}$ still impacts discomfort, but less than $D_{\text {vert }}$. As already shown in Table 6, the weighted RMS for longitudinal acceleration is zero in all simulations, therefore $D_{\text {lon }}$ is constant. The discomfort due to roll acceleration $D_{\text {roll }}$ is lower than due to pitch acceleration $D_{\text {pitch }}$ across all simulations.

Simulations for Test Case 1 and 2 are conducted with lower aircraft mass than for Test Case 3 and 4 . With more aircraft mass, the aircraft is significantly less accelerated in turbulent flight resulting in lower discomfort values as one may expect. The c.g. position seemingly only affects $D_{\text {vert }}$ and $D_{\text {pitch. }}$.

The GLA mode only impacts $D_{\text {vert }}$ and $D_{\text {pitch. Table } 8}$ shows the relative difference of GLA disabled (OFF) and GLA enabled (ON) discomfort results in all four test cases, with the GLA OFF results as the reference values. With GLA ON, the discomfort values for $D_{\text {vert }}$ and $D_{\text {pitch }}$ reduce by approximately $5-7 \%$ and $25-60 \%$ respectively.
Table 7 Discomfort factors for the single axis

\begin{tabular}{lllllll}
\hline TC & GLA & $D_{\text {vert }}$ & $D_{\text {lat }}$ & $D_{\text {lon }}$ & $D_{\text {roll }}$ & $D_{\text {pitch }}$ \\
\hline 1 & OFF/ & 0.66 & 0.15 & -0.02 & 0.02 & 0.18 \\
& ON & 0.62 & 0.15 & -0.02 & 0.02 & 0.13 \\
2 & OFF/ & 0.68 & 0.15 & -0.02 & 0.02 & 0.17 \\
& ON & 0.63 & 0.15 & -0.02 & 0.02 & 0.12 \\
3 & OFF/ & 0.38 & 0.09 & -0.02 & 0.01 & 0.06 \\
& ON & 0.36 & 0.09 & -0.02 & 0.01 & 0.02 \\
4 & OFF/ & 0.38 & 0.09 & -0.02 & 0.01 & 0.10 \\
& ON & 0.35 & 0.09 & -0.02 & 0.01 & 0.07 \\
\hline
\end{tabular}


Table 8 Relative difference (GLA OFF/ON) for discomfort factors for single axes

\begin{tabular}{lll}
\hline TC & $D_{\text {vert }}(\%)$ & $D_{\text {pitch }}(\%)$ \\
\hline 1 & -6.56 & -26.14 \\
2 & -7.06 & -25.66 \\
3 & -5.50 & -59.70 \\
4 & -6.30 & -32.14 \\
\hline
\end{tabular}

Table 9 Discomfort factors for combined axis

\begin{tabular}{lllll}
\hline TC & GLA & $D_{\mathrm{VLR}}$ & $D_{\mathrm{LP}}$ & $D_{\mathrm{VIB}}$ \\
\hline 1 & OFF/ & 0.70 & 0.09 & 0.71 \\
& ON & 0.66 & 0.05 & 0.66 \\
2 & OFF/ & 0.72 & 0.09 & 0.73 \\
& ON & 0.67 & 0.05 & 0.68 \\
3 & OFF/ & 0.39 & 0.02 & 0.39 \\
& ON & 0.37 & 0.01 & 0.36 \\
4 & OFF/ & 0.39 & 0.03 & 0.39 \\
& ON & 0.37 & 0.02 & 0.36 \\
\hline
\end{tabular}

Table 9 shows the absolute discomfort values for the combined axis for all test cases with GLA off and on. The discomfort contribution for the combined axes of vertical, lateral and roll vibrations $D_{\mathrm{VLR}}$ is a much bigger contributor to discomfort than the discomfort contribution for the combined axes of longitudinal and pitch vibrations $D_{\mathrm{LP}}$ with both GLA settings. Due to the difference in magnitude between $D_{\mathrm{VLR}}$ and $D_{\mathrm{LP}}$, the overall discomfort value $D_{\mathrm{VIB}}$ has almost in all cases the same value as $D_{\mathrm{VLR}}$. The discomfort factors are affected by the c.g. position and are lower with increasing aircraft mass, as already discussed for the single axes discomfort contributions. With enabled GLA, $D_{\mathrm{VIB}}$ reduces by $6.0-7.3 \%$ depending on the test case. The main contribution comes from discomfort due to vertical, lateral and roll accelerations $D_{\mathrm{VLR}}$, as its magnitude is much higher than $D_{\text {LP. }}$

To summarize the results, the implemented GLA has a positive impact on passenger comfort in all investigated test cases. According to Fig. 12, the percentage of passengers who would find the ride uncomfortable e.g. in Test Case 1 would be approx. $32 \%$ with GLA enabled instead of approx. $35 \%$ with GLA disabled.

\section{Conclusion}

Future aircraft designs will be more flexible and use active loads functions, which can influence the aircraft rigid-body motion. It is important to evaluate the impact of structural design choices including gust load alleviation functions on flight mechanics as early as possible to reduce development risks. Currently used semi-empirical methods do not allow such detailed multi-disciplinary assessments in early design. That is why TUB and DLR contributed the described toolchain in this work to gain early knowledge about the structural and flight mechanical interdependencies. This work demonstrates the toolchain by investigating passenger ride comfort in turbulent flight. Without and with gust load alleviation, the largest contribution to discomfort comes from the translational acceleration in vertical direction followed by the angular acceleration in lateral and pitch direction. The acceleration in longitudinal and roll direction are negligible in comparison.

Gust load alleviation reduces the acceleration acting on passengers in vertical direction and the pitch axis, although it is not explicitly designed to lower accelerations, which can be achieved e.g. with mode damping features using acceleration feedback. That is why it can be said that for this particular aircraft configuration, GLA reduces loads and improves passenger ride comfort at the same time. Furthermore, this investigation opens the possibility to explore ride comfort potentials of GLA further with relatively little effort. In the described case, a simple feed-forward GLA which is designed to reduce loads during 1-cos gust encounters is taken into account. A possible next step would be to optimize the GLA both for load reduction during gust encounters and ride comfort improvement, e.g. with various prioritization of both objectives. Such investigations are assumed to be easier to conduct in early design stages, where the aircraft design is not finalized.

This work shows that the proposed toolchain is suitable for complex, fast and comprehensive flight mechanical studies, while having a structural model which is in tune with the design loads. The toolchain runs on conventional desktop computer hardware and many steps within the toolchain are already automated, which reduces time required for assessments. Eventually, such studies broadens this design space for more flexible aircraft and reduce development risks. In its current form, the toolchain only allows assessments of models with linear deformation. On one hand, there is indeed research demand regarding large, nonlinear deformations. On the other hand, the proposed toolchain rather provides a multidisciplinary characteristics since it considers a flexible structure, loads and structural optimization, control theory, flight mechanics and passenger.

As an outlook, TUB will enhance the simulation model and include more acceleration measurement points and the calculation of the wing root bending moment during simulations. This will allow to investigate flight mechanical requirements and critical loads simultaneously in flight simulations. This is necessary when assessing more complex active control algorithms, e.g. those using feedback loops. 
Acknowledgements The work presented in this report was funded by the German Federal Ministry for Economic Affairs and Energy (BMWi) due to resolution of the German Federal Parliament within the scope of the LuFo V/5 project CON.MOVE (Grant number for TUB 20A1505D and for DLR 20A1505C).

Funding Open Access funding enabled and organized by Projekt DEAL. German Federal Ministry for Economic Affairs and Energy (BMWi).

Availability of data and material Not applicable.

Code availability Not applicable.

\section{Declarations}

Conflict of interest The author(s) declare no competing interests.

Open Access This article is licensed under a Creative Commons Attribution 4.0 International License, which permits use, sharing, adaptation, distribution and reproduction in any medium or format, as long as you give appropriate credit to the original author(s) and the source, provide a link to the Creative Commons licence, and indicate if changes were made. The images or other third party material in this article are included in the article's Creative Commons licence, unless indicated otherwise in a credit line to the material. If material is not included in the article's Creative Commons licence and your intended use is not permitted by statutory regulation or exceeds the permitted use, you will need to obtain permission directly from the copyright holder. To view a copy of this licence, visit http://creativecommons.org/licenses/by/4.0/.

\section{References}

1. Roskam, J.: Airplane Design VII: Determination of Stability, Control and Performance Characteristics: FAR and Military Requirements. DARcorporation, Lawrence (1985). ISBN 978-1-88488-554-9

2. Klimmek, T., Schulze, M., Abu-Zurayk, M., Ilic, C., Merle, A.: CPACS-MONA - an independent and high-fidelity based MDO tasks integrated process for the structural and aeroelastic design of aircraft configurations. In: Presented at IFASD 2019, Savannah, USA

3. Krishnamurthy, V., Luckner, R.: Automated evaluation of handling qualities and performance for preliminary aircraft design using flight simulation models. In: Presented at DGLR 2014, Augsburg, Germany. isbn:urn:nbn:de:101:1-201511134293

4. Kaiser, C., Friedewald, D., Nitzsche, J.J: Comparison of nonlinear CFD with time-linearized CFD and CFD-corrected DLM for gust encounter simulations. Presented at IFASD 2017, Como, Italy. ISBN:978-88-97576-28-0

5. Quero, D.: An aeroelastic reduced order model for dynamic response prediction to gust encounters. Dissertation, TU Berlin, Germany, DLR Research Report DLR-FB-2017-36 (2017)

6. Handojo, V., Lancelot, P., De Breuker, R.: Implementation of active and passive loads alleviation methods on a generic midrange aircraft configuration. In: Presented at AIAA Aviation 2018, Atlanta, USA. https://doi.org/10.2514/6.2018-3573
7. EASA.: Certification Specifications and Acceptable Means of Compliance for Large Aeroplanes CS-25. EASA, Cologne (2019)

8. Klimmek, T.: Statische aeroelastische Anforderungen beim multidisziplinären Strukturentwurf von Verkehrsflugzeugflügeln. Dissertation, Technische Universität Braunschweig, Germany (2016)

9. Handojo, V.: Contribution to load alleviation in aircraft pre-design and its influence on structural mass and fatigue. Dissertation, TU Berlin, Germany, tbp

10. König, R., Hahn, K.: Load alleviation and ride smoothing investigations using ATTAS. In: Proceeding of the 17th ICAS, Stockholm, Sweden (1990)

11. US Department of Defense: Flying Qualities of Piloted Airplanes-MIL-F-8785C. US Department of Defense, USA (1980)

12. US Department of Defense: Flying Qualities of Piloted AircraftMIL-STD-1797A. US Department of Defense, USA (2004)

13. Krishnamurthy, V., Luckner, R.: Flight mechanical modeling considering flexibility and flight control functions in preliminary aircraft design. In: Presented at AIAA Aviation 2017, Denver, USA. https://doi.org/10.2514/6.2017-4332

14. Krishnamurthy, V., Hamann, A., Luckner, R.: Tool chain for model-based flight mechanical evaluation in preliminary aircraft design for commercial transport aircraft with electronic flight control systems. Presented at CEAS 2017, Warsaw, Poland

15. Krishnamurthy, V., Hamann, A., Luckner, R.: Prozesskette, Automatisierte, zur flugmechanischen Bewertung von Parameteränderungen im Rahmen des modellbasierten Flugzeugvorentwurfs. Presented at DGLR, Rostock, Germany. urn:nbn:de urn:nbn:de:101:1-201601222820 (2014)

16. McDonnell Douglas Astronautics Company.: USAF Stability and Control DATCOM-AFWAL-TR-83-3048. McDonnell Douglas Astronautics Company, New York (1978)

17. Silvestre, F.J.: Methodology for modeling the dynamics of flexible, high-aspect-ratio aircraft in the time domain for aeroservoelastic investigations. Dissertation, Technische Universität Berlin, mensch und buch verlag, Germany (2013). ISBN:978-3-86387-316-5

18. Fezans, N., Joos, A.H.-D.: Combined feedback and LIDAR-based feedforward active load alleviation. In: Presented at AIAA Aviation 2017, Denver, USA. https://doi.org/10.2514/6.2017-3548

19. Leatherwood, J.D., Dempsey, T.K., Clevenson, S.A.: A Design Tool for Estimating Passenger Ride Discomfort Within Complex Ride Environments. Human Factors, New York (1980). https:// doi.org/10.1177/001872088002200304

20. Leatherwood, J.D., Barker, L.M.: A user oriented and computerized model for estimating vehicle ride quality. NASA Technical Paper 2299, USA (1984)

21. Natke, H.G.: Einführung in Theorie und Praxis der Zeitreihenund Modalanalyse. Friedr. Vieweg und Sohn, Germany (1983). ISBN 978-3-322-94266-1

22. Diederick, M.: Savitzky-Golay Smooth-Differentiation Filters and Filter Application, Mathwork Matlab - File Exchange, 2019. [Online; posted 03-09-2019]. https://www.de.mathworks.com/ matlabcentral/fileexchange/30299-savitzky-golay-smooth-Diffe rentiation-filters-and-filter-application. Accessed 28 June 2020

Publisher's Note Springer Nature remains neutral with regard to jurisdictional claims in published maps and institutional affiliations. 